\title{
Seismic assessment of buildings by rapid visual screening procedures
}

\author{
P. Kapetana \& S. Dritsos \\ Department of Civil Engineering, University of Patras, Patras, Greece
}

\begin{abstract}
Recently, several pre-earthquake screening methods have been developed in order to rapidly evaluate the vulnerability profile of the existing building stock, which has been constructed before or after the adoption and enforcement of seismic codes. The objective of these methods is to identify, inventory and rank all high-risk buildings in a specified region so that a strategy of priority based interventions to buildings can be formed. Major parameters that have affects on the seismic risk are the seismicity of the location, vulnerability and importance of the building structure. The most known rapid visual screening methods have been developed in countries of high seismic risk such as the USA, Greece, New Zealand, India and Canada and they are briefly described in this paper. Furthermore, these methods are applied to a sample of 456 reinforced concrete buildings, located in Athens, whose structural characteristics and levels of damage by the 1999 Athens earthquake are known. In particular, 93 buildings collapsed, 201 sustained severe damage, 69 moderate and 93 buildings sustained light damage. By the methods' implementation, eight different scores have been determined for each building, according to the scoring systems of the applied methods. The results of those applications are used to evaluate the methods' reliability in identifying potentially seismically hazardous reinforced concrete buildings. The obtained results indicate that the implementation of the Greek method results in the most reasonable connection between damage severity and structural scores for all levels of damage, while the Greek method is represented to be the most efficient in terms of both predicting the damage level and leading to the reliable formation of a high-priority set of buildings.
\end{abstract}

Keywords: pre-earthquake, rapid, visual, screening, seismic, vulnerability, assessment, building. 


\section{Introduction}

Worldwide, several empirical screening methods have been developed which can help to rapidly evaluate the vulnerability profile of large number of different types of buildings. Pre-earthquake screening of buildings is already used in many earthquake prone countries to identify the most potentially seismically hazardous buildings and prioritizing the ones that would warrant a more detailed analysis. Pre-earthquake screening methods can be divided into two primary categories. The first one concerns methods whose implementation requires both rapid visual screening of the building and determination of a structural score and the second one concerns methods whose implementation requires measuring out some dimensions of the construction and performing some simple structural calculations. The most known rapid visual screening methods that have been worldwide proposed are: the method of the U.S.A by the Federal Emergency Management Agency (FEMA), the Greek method developed by the Earthquake Planning and Protection Organization (OASP), Rapid Evaluation Method by the New Zealand Society for Earthquake Engineering (NZSEE), India's method by the Indian Institute of Technology and the used methodology in Canada developed by the National Research Council's (NRC) Institute for Research in Construction. To the second category belong: the Japanese method developed by the Japanese Building Disaster Prevention Association (JBDPA), the Turkish method developed by the Structural Engineering Research Unit, the Initial Evaluation Process by the New Zealand Society for Earthquake Engineering and the Italian method by the National Earthquake Defense Group (GNDT). The methods used and applied in this paper belong to the first category, while the main aim is to ascertain which rapid visual screening method seems to be the most credible.

\section{Rapid visual screening methods}

Rapid visual screening entails assessing buildings to ascertain their level of seismic risk following a simplified procedure whose main objective is to determine if the buildings should or should not be subject to a more detailed investigation. Particularly, these procedures include completing special Data Collection Forms concerning structural and non-structural characteristics of the construction and determining a Structural Score according to which construction gets ranked.

\subsection{The method of the U.S.A. by the Federal Emergency Management Agency}

The procedure for Rapid Visual Screening (RVS) was first proposed in the U.S.A and was given in "Rapid Visual Screening of Buildings for Potential Seismic Hazards: A Handbook" in 1988. The procedure was further modified in 2002 to incorporate latest technological advancements and lessons from earthquake disasters in the 1990s. Even though this RVS procedure was 
originally developed for typical constructions in the U.S.A, it has been widely used in many other countries after suitable modifications. According to the last edition, the first step of the RVS process includes identification of the primary structural lateral load-resisting system and classification of the building in one of 15 structural type categories, according to the building's material. By this classification, the building gets a basic score, which then gets modified due to probable vulnerability attributes concerning the building's shape (irregularities, high rise, soft story, torsion, short columns, large heavy cladding) and soil conditions. Should the final Structural Score be lower than 2, then a more detailed analysis is required. The inspection, data collection and decision-making process typically occurs at the building site and is expected to take around 30 minutes for each building (FEMA, [4]). It should be noted that, within the framework of this paper, the method of the first edition will be called FEMA-88 and that of the second one FEMA-02.

\subsection{The Greek method by the Earthquake Planning and Protection Organization}

The Greek method developed by OASP in 2000 is based on the first edition of the FEMA 154 Handbook and will be called OASP-0. The method provides a standard rapid visual screening procedure to identify both the primary structural lateral-load-resisting system and structural materials of the building. By this identification, the building gets classified in one of 18 structural types and it is awarded an Initial Structural Hazard Score. Then, this score will be modified by identifying both the seismic zone and three significant structure characteristics (weak story, short columns and regular arrangement of the masonry) that affect the building's seismic response to arrive at the Basic Structural Hazard Score. Finally, this score will be modified by identifying some modifiers related to the observed performance attributes to arrive at the Final Score. Buildings having a Final Score of 2 or less should be investigated in more detail (OASP, [3]). However, since hazard scores and score modifiers are in question, two alternative scoring scenarios have been proposed in order to identify potentially hazardous buildings more accurately. The first one is based on the OASP-0 method and denoted will be in the following OASP-R and the second one is based on the second edition of the FEMA 154 Handbook and will be denoted FEMA-G.

\subsection{Rapid evaluation method by the New Zealand Society for Earthquake Engineering}

The Rapid Evaluation, proposed in 1996, largely follows the process presented in the first edition of the FEMA 154 Handbook with the significant variation away from presenting a relatively abstract score, which reflects the logarithm of probability of a damage state, to presenting a score, which has a loose and very conservative relationship to a damage ratio. Differences between FEMA and NZSEE methods concern the number of structural types and the score modifiers considered, while the request for a more detailed evaluation of the building 
comes from a graph, which is a function of the building gross area and the final structural score (Brundson et al, [1]). In the following the methodology by the NZSEE will be denoted as NZ-96.

\subsection{Seismic vulnerability assessment of buildings in India}

The procedure for Rapid Visual Screening used in India is similar to that developed by the FEMA in 2002 after suitable modifications. The modifications concern both soil and structural types and the values of the considered modifiers. Particularly, there are three soil and ten structural types considered (no tilt-up or reinforced masonry buildings are included). Generally, the final score $\mathrm{S}<0.7$ indicates high vulnerability requiring further evaluation and retrofitting of the building (Sinha and Goyal, [6]).

\subsection{Seismic screening of buildings in Canada}

The widely used methodology in Canada is given in the "Manual for Screening of Buildings for Seismic Investigation" in 1993 and it was developed by the NRC. Its purpose is to establish numerically a Seismic Priority Index (SPI)-a ranking-which results from the addition of a Structural Index and a NonStructural Index. Major factors in determining the screening score are the building location, soil conditions, type and use of the structure, obvious building irregularities, the presence or absence of non-structural damages, building age and the building importance and occupancy characteristics. Should SPI be greater than 20 the priority is evaluated to be high (CCIPEP, [2]).

\section{Implementation of alternative rapid visual screening methods}

As it has been mentioned, within the framework of this paper, it is attempted to ascertain the efficiency of the presented rapid visual screening methods in identifying potentially hazardous buildings. Thus, a database has been used, which had been created shortly after the 1999 Athens earthquake (Karabinis, [5]). The database consists of 456 reinforced concrete buildings, located in N.W. of Athens and constructed between 1950 and 2000, whose structural characteristics and levels of damage by the 1999 Athens earthquake are known. In particular, 93 buildings collapsed, 201 buildings sustained severe damage, 69 buildings moderate damage and 93 buildings sustained light damage. The seismicity level of the area is moderate (seismic zone II), while soil conditions are of type A (rock or rigid clay). According to the scoring systems of the presented methods (OASP-0, OASP-R, FEMA-02, FEMA-G, FEMA-88, INDIA, NZ-96, CANADA), eight different structural scores have been determined for each building and the results of the methods' implementation are given below. 


\subsection{Percentages of buildings per set of structural scores}

Figures 1-4 illustrate the correlation between percentages of buildings and structural scores, as they have been determined by the scoring systems of the applied methods, for all damage categories (collapsed buildings, buildings with severe, moderate and light damage). In addition, both the trendlines of the graphs, which are graphic representations of trends in data series and come from linear regression analysis, and the corresponding coefficients of determination $\mathrm{R}^{2}$.
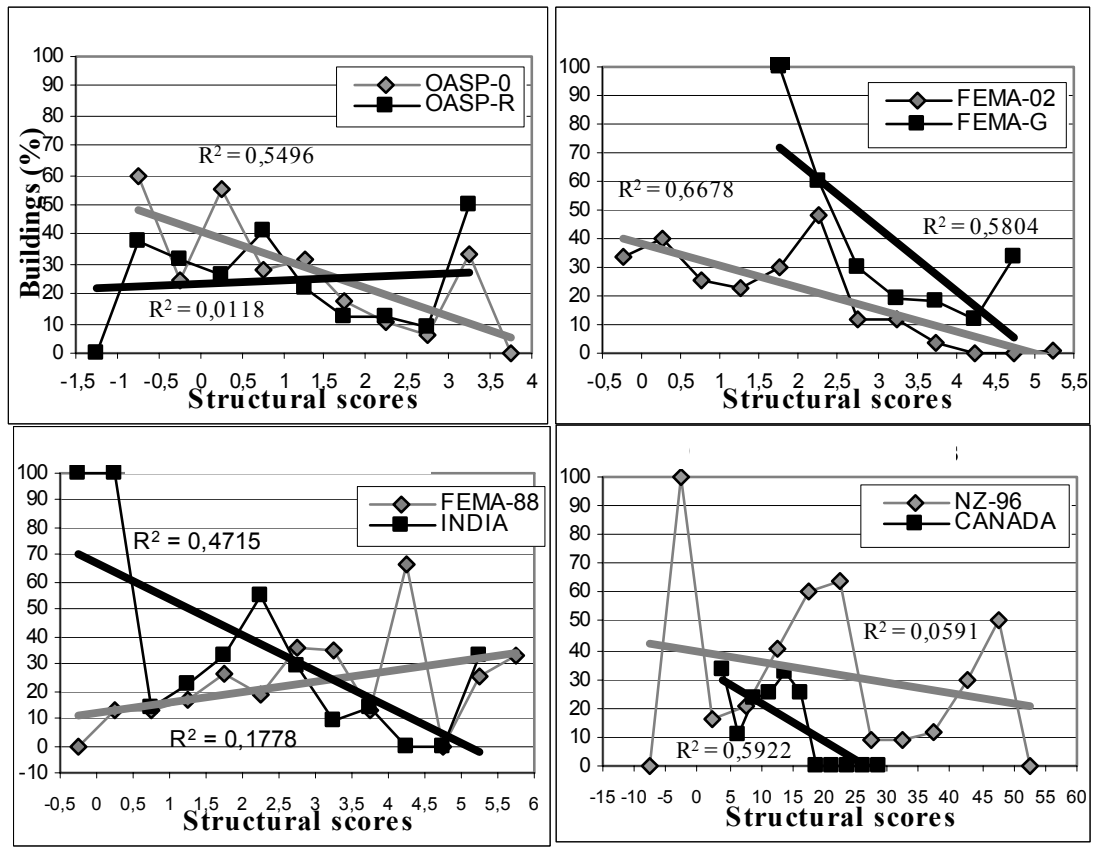

Figure 1: Collapsed buildings.

From the graphs of Fig.1, it is observed that, concerning the category of collapsed buildings, the implementations of OASP-0, FEMA-02, FEMA-G and India's method result in a reasonable connection between the number of the collapsed buildings and structural scores, because as structural scores increase percentages of collapsed buildings decrease. On the contrary, the implementations of OASP-R and FEMA- 88 methods result in an unreasonable connection between damage severity and structural scores, because as structural scores increase percentages of collapsed buildings increase. The same conclusion is obtained for the NZ-96 and Canada methods, since in the above scoring systems high values of scores indicate high vulnerability.

From Figure 2, it can be seen that FEMA-02, Indian, FEMA-G and Canada methods are ineffective in predicting severe damages, while FEMA-88's precision in representing possible severe damage is the highest one. 

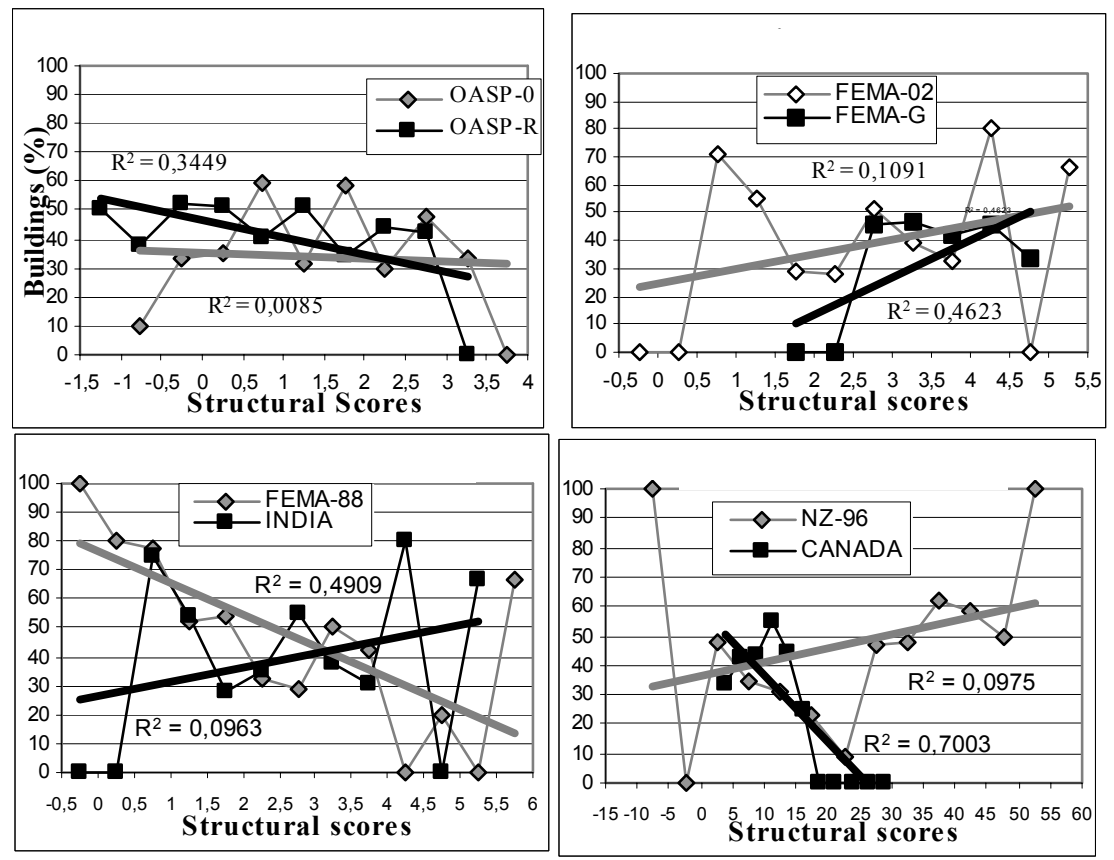

Figure 2: Buildings with severe damage.
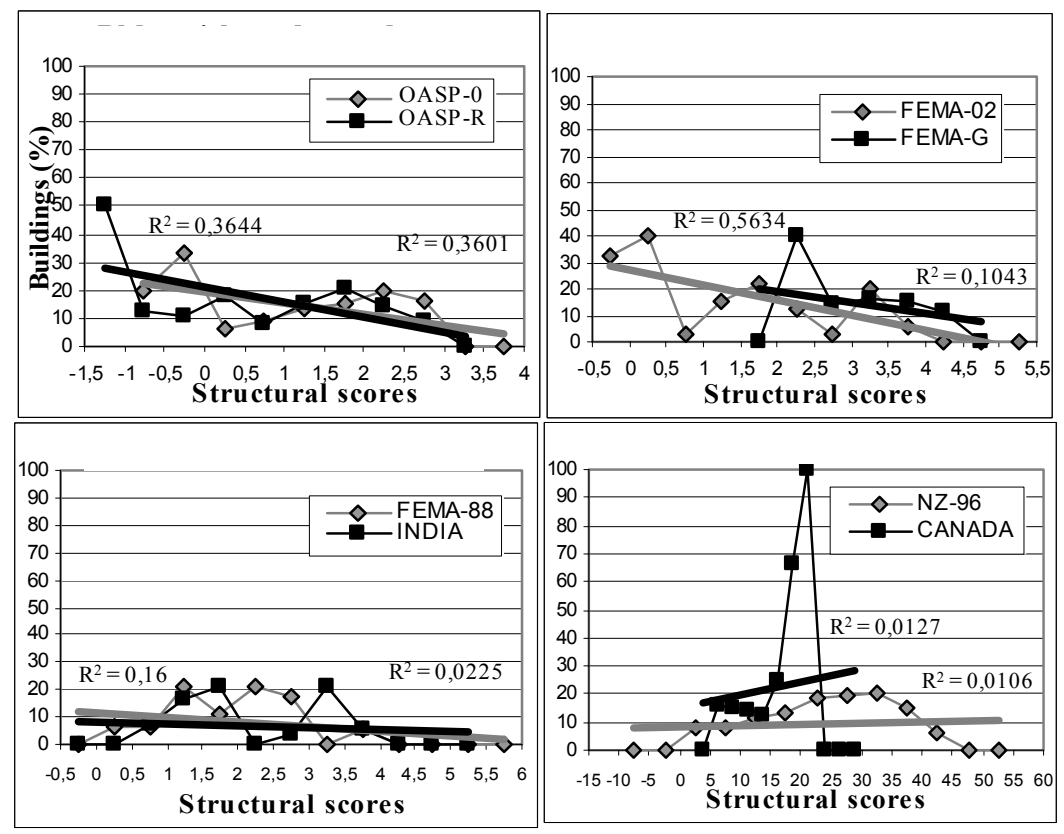

Figure 3: Buildings with moderate damage. 
Figure 3 represents that buildings with moderate damage are identified as such by all rapid visual screening methods, with FEMA-02 predicting possible moderate damage more effectively than the other methods do.

Concerning buildings with light damage, it can be seen that FEMA-02 and Canada methods are not effective in identifying buildings safe in earthquake, while the other methods are presented to be quite reliable in predicting potential light damage.
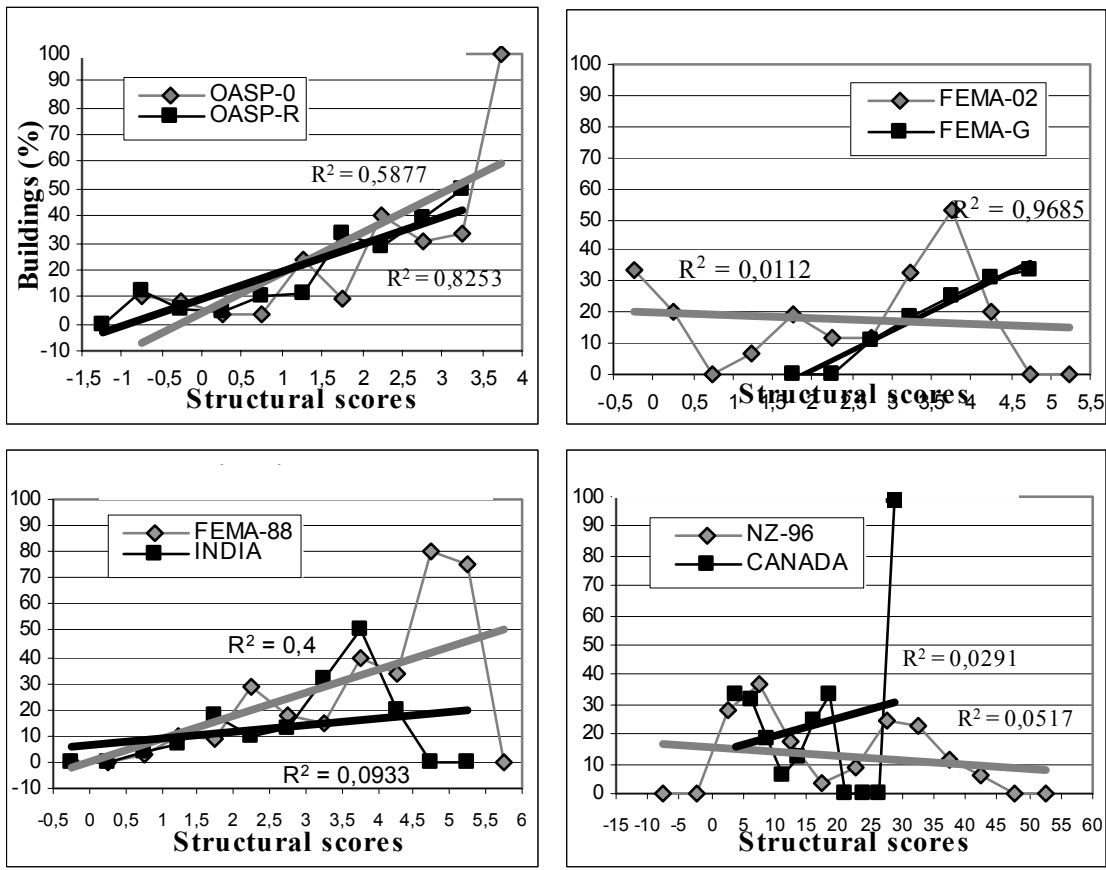

Figure 4: Buildings with light damage.

\subsection{Averages of structural scores per building damage category}

Figure 5 shows the averages of structural scores per building damage category for all rapid visual screening methods. Collapsed buildings are indicated with "C", buildings with severe damage with "S", buildings with moderate damage with " $M$ " and buildings with light damage with " $L$ ". It is observed that for OASP-0, OASP-R, FEMA-02 and the Indian method, there is a reasonable connection between values of averages, because as damage gets more severe so averages decrease. A similar tension is observed for FEMA-G and FEMA-88 methods. However, the results are not that satisfying, because, concerning FEMA-88 method, collapsed buildings have an average of structural scores greater than buildings with severe damage do and, concerning FEMA-G method, buildings with severe damage have an average of structural scores greater than buildings with moderate damage do. Finally, among NZ-96 average values an 
unreasonable connection is presented, while Canada averages seem to be in agreement with damage severity, apart from buildings with moderate damage that have an average greater than buildings with severe damage do.
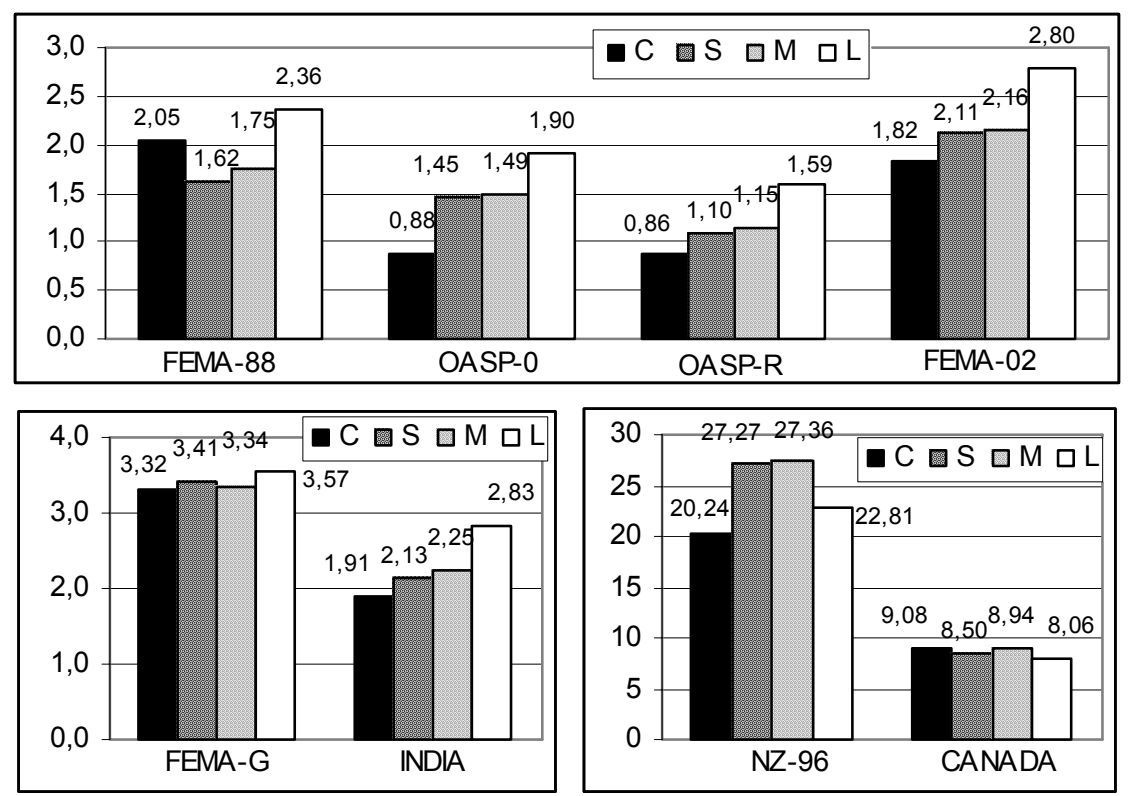

Figure 5: Averages of structural scores per building damage category.

Table 1: $\quad$ Methods' effectiveness.

\begin{tabular}{|c|c|c|c|c|c|c|c|c|}
\hline \multirow{3}{*}{ Method } & \multicolumn{8}{|c|}{ BUILDING DAMAGE CATEGORIES } \\
\hline & \multicolumn{2}{|c|}{ Collapse } & \multicolumn{2}{|c|}{$\begin{array}{c}\text { Severe } \\
\text { damage }\end{array}$} & \multicolumn{2}{|c|}{$\begin{array}{c}\text { Moderate } \\
\text { damage }\end{array}$} & \multicolumn{2}{|c|}{ Light damage } \\
\hline & [1] & {$[2]$} & [1] & [2] & [1] & [2] & [1] & [2] \\
\hline OASP-0 & $\checkmark$ & $\checkmark$ & $\checkmark$ & $\checkmark$ & $\checkmark$ & $\checkmark$ & $\checkmark$ & $\checkmark$ \\
\hline OASP-R & $x$ & $\checkmark$ & $\checkmark$ & $\checkmark$ & $\checkmark$ & $\checkmark$ & $\checkmark$ & $\checkmark$ \\
\hline FEMA-02 & $\checkmark$ & $\checkmark$ & $x$ & $\checkmark$ & $\checkmark$ & $\checkmark$ & $x$ & $\checkmark$ \\
\hline FEMA-G & $\checkmark$ & $\checkmark$ & $x$ & $x$ & $\checkmark$ & $\checkmark$ & $\checkmark$ & $\checkmark$ \\
\hline FEMA-88 & $x$ & $x$ & $\checkmark$ & $\checkmark$ & $\checkmark$ & $\checkmark$ & $\checkmark$ & $\checkmark$ \\
\hline INDIA & $\checkmark$ & $\checkmark$ & $x$ & $\checkmark$ & $\checkmark$ & $\checkmark$ & $\checkmark$ & $\checkmark$ \\
\hline NZ-96 & $x$ & $x$ & $\checkmark$ & $x$ & $\checkmark$ & $x$ & $\checkmark$ & $\checkmark$ \\
\hline CANADA & $x$ & $\checkmark$ & $x$ & $\checkmark$ & $\checkmark$ & $x$ & $x$ & $\checkmark$ \\
\hline
\end{tabular}

In order to reach conclusions relative to methods' efficiency, the results obtained by reviewing Figures 1-5 were summarized and they are presented in Table 1. Columns indicated with (FEMA, [4]) concern the evaluation results 
obtained from paragraph 3.1, while those with concern the evaluation results from paragraph 3.2. Method's effectiveness is indicated with " $\checkmark$ " and ineffectiveness with " $x$ ".

It is observed that OASP-0 method is the only one effective in identifying reinforced concrete buildings both safe and not safe in earthquake in both evaluation tasks.

\subsection{Efficiency measure of collapse prediction}

An alternative criterion for the methods' efficiency would be the comparison of the percentages of buildings that did actually collapse in several high priority sets.

Table 2: $\quad$ Efficiency measures.

\begin{tabular}{|c|c|c|c|}
\hline Subset & $10 \%$ of bldgs $=46$ & $20 \%$ of bldgs $=92$ & $50 \%$ of bldgs $=228$ \\
\hline Method & $\mathrm{E}_{\mathrm{C}, 10}$ & $\mathrm{E}_{\mathrm{C}, 20}$ & $\mathrm{E}_{\mathrm{C}, 50}$ \\
\hline OASP-0 & 0.57 & 0.46 & 0.33 \\
\hline OASP-R & 0.46 & 0.42 & 0.28 \\
\hline FEMA-02 & 0.39 & 0.38 & 0.29 \\
\hline FEMA-G & 0.28 & 0.27 & 0.26 \\
\hline FEMA-88 & 0.11 & 0.18 & 0.27 \\
\hline INDIA & 0.48 & 0.34 & 0.29 \\
\hline NZ-96 & 0.12 & 0.26 & 0.13 \\
\hline CANADA & 0.16 & 0.27 & 0.29 \\
\hline
\end{tabular}

As these percentages indicate efficiency towards collapse prediction, the efficiency measure of collapse prediction, $\mathrm{E}_{\mathrm{C}}$, can be adopted for their reference. Obviously, the higher this percentage is, the more efficient the method can be considered. Table 2 lists the efficiency measures of collapse prediction that correspond to $10 \%, 20 \%$ and $50 \%$ high priority subsets for all methods, denoted as $\mathrm{E}_{\mathrm{C}, 10}, \mathrm{E}_{\mathrm{C}, 20}$ and $\mathrm{E}_{\mathrm{C}, 50}$ respectively. It is observed that OASP-0 method is characterized by the highest measures in all subsets examined, while Indian, OASP-R and FEMA-02 methods follow. Unlike, FEMA-88 and NZ-96 methods are characterized, in general, by the lowest measures. However, when $50 \%$ high priority set is examined, it can be seen that, except from NZ-96, measure values of all methods are very close. Suppose that there would be available budget for detailed analysis only for $10 \%$ high priority set of buildings that are $0.10 \times 456=46$ buildings. If those buildings were to be selected prior to the earthquake strike by chance, without using the results of some rapid visual screening procedure, then in this set would be included $0.10 \times 93=9.3$ buildings that would finally collapse. Unlike, if buildings' selection had been based on the results obtained by using OASP- 0 method, then $0.57 \times 46=26$ buildings would be prevented from collapsing. It should be noted that a measure of $93 / 456=0.2$ or less would not be acceptable, because that would indicate that the method's 
efficiency in collapse prediction is not reliable and that selecting buildings for further investigation by chance would prevent an equal or greater number of buildings from collapse.

\section{Conclusions}

Assessing the results from the implementation of rapid visual screening methods, the following conclusions are reached: (a) A reasonable correlation between structural scores and collapse probability appears to exist only when scoring systems of OASP-0, FEMA-02, Indian and FEMA-G methods are used, (b) The averages of structural scores per building damage category have a reasonable connection with damage severity only when OASP-0, OASP-R, FEMA-02 and the Indian method are implemented. In addition, OASP-0 method appears to have the best scoring difference between averages of collapsed buildings and buildings with little damage, (c) OASP-0, OASP-R, Indian and FEMA-02 methods are characterized by the highest efficiency measures of collapse prediction when $10 \%, 20 \%$ and $50 \%$ high priority subsets are examined, with the OASP-0 measure being the highest of all. However, for $50 \%$ priority subsets, values of measures are almost the same, apart from that of New Zealand.

The reached conclusions above come from a limited number of data, related to the seismic response of existing buildings in earthquake. Thus, in order to propose the most reasonable rapid evaluation procedure, the assessment of additional data is required.

\section{References}

[1] Brundson, D., Holmes, S., Hopkins, D., Merz, S., Jury, R. \& Shephard, B., Rapid Evaluation (Chapter 4). The Assessment and Improvement of the Structural Performance of Earthquake Risk Buildings. Report. Draft for General Release for the Building Industry Authority by the New Zealand Society for Earthquake Engineering, pp. 22-35, 1996.

[2] Canadian Office of Critical Infrastructure Protection and Emergency Preparedness (CCIPEP), Seismic Hazard, Building Codes and Mitigation Options for Canadian Buildings, www.ocipep-bpiepc.ca

[3] Earthquake Planning and Protection Organization (OASP). Provisions for Pre-Earthquake Vulnerability Assessment of Public Buildings (Part A), Athens, Greece, 2000. (In Greek).

[4] Federal Emergency Management Agency (FEMA). Rapid Visual Screening of Buildings for Potential Seismic Hazards: A Handbook, ATC: Redwood City, California, 1988, $2^{\text {nd }}$ Edition, 2002.

[5] Karabinis, A. Rating of the First Level of Pre-Earthquake Assessment, Final Report, Earthquake Planning and Protection Organization, Athens, Greece, 2004. (In Greek).

[6] Sinha, R. \& Goyal, A. A National Policy for Seismic Vulnerability Assessment of Buildings and Procedure for Rapid Visual Screening of Buildings for Potential Seismic Vulnerability, Published by the Indian Institute of Technology, Dept. of Civil Engineering, Bombay, 2002. 